\title{
Percepción del blended learning en profesores y estudiantes universitarios
}

\section{Perception of blended learning in professors and university students}

\author{
Ma. del Carmen Nolasco Salcedo \\ ORCID: 0000-0002-5903-3929/mcns08@gmail.com \\ Profesora-investigadora, Centro Universitario de La Ciénaga (CUCI), Universidad de Guadalajara (UDG)
}

\section{Luis Mexitli Orozco Torres}

ORCID: 0000-0001-6576-9767/mexitli.orozco@cuci.udg.mx

Profesor-investigador, CUCI, UDG

\section{RESUMEN}

El blended learning (o b-learning, "aprendizaje combinado") es una modalidad de enseñanza-aprendizaje con una amplia gama de diversas técnicas que emerge de los espacios virtuales, de la evolución de nuevas formas de enseñar, de la apertura al cambio y de la innovación de estrategias de aprendizaje. La continua evolución en las tecnologías de la información y comunicación (TIC) ha contribuido a una educación más dinámica, eficiente y flexible. Esta investigación presenta resultados y experiencias de docentes y discentes, con énfasis en el uso del modelo educativo del $b$-learning, lo que permite al discente desarrollar el pensamiento crítico-reflexivo-constructivo para la resolución de problemas, además del desarrollo de competencias de estudiantes y docentes, lo que facilita la construcción de un aprendizaje significativo.

\section{PALABRAS CLAVE}

blended-learning, docentes, estudiantes, ambientes virtuales, aprendizaje invertido
ABSTRACT

Blended learning (b-learning) is a teaching-learning method with a wide range of different techniques that emerges from online education, from the evolution of new ways of teaching, from the opening to change and from the innovation of learning strategies. The continuous evolution in information and communication technologies (ICT) has contributed to a more dynamic, efficient and flexible education. This research shows results and experiences of professors and students, with emphasis on the use of the educational model of b-learning, which allows the student to develop critical-reflective-constructive thinking for problem solving, in addition to the development of skills in both students and professors, which facilitates the construction of meaningful learning.

\section{KEY WORDS}

Blended-Learning, teachers, students, virtual environments, flipped learning 


\section{Introducción}

En los últimos años, con el continuo desarrollo de las tecnologías de la información y la comunicación (TIC), la modalidad educativa b-learning (blended learning, "aprendizaje combinado") surge como el método de instrucción más destacado en la educación, especialmente en la educación superior. El entorno tradicional en el que se lleva a cabo el proceso de aprendizaje, de forma presencial, independientemente de la intensidad con la que se utilice la tecnología, tiene algunas restricciones importantes. Algunas de estas restricciones son la interacción limitada entre profesor y alumno. Entonces, un factor importante es desarrollar cursos utilizando b-learning para ofrecer una opción viable a los estudiantes que buscan la flexibilidad de los cursos sólo a distancia, pero que también desean tener algún contacto personal con el profesorado y otros estudiantes en el aula. El proceso de enseñanza-aprendizaje utilizando la modalidad b-learning va más allá de las barreras de tiempo, ubicación y cultura y ha creado muchas oportunidades mejoradas para discentes y docentes.

La metamorfosis que ha estado sufriendo la educación en la última década ha dejado constancia de la necesidad de adaptarse a las diversas herramientas que proporcionan las TIC en todos los niveles. La globalización de las tecnologías está insertada en cada rincón de las instituciones educativas: en las aulas, en la cafetería, en los jardines, entre otros, y transforma el concepto de la educación, impulsado por la revolución tecnológica, donde los estudiantes son los protagonistas de su formación, basada en el éxito de una educación inteligente.

El $b$-learning es un modelo que permite potenciar y combinar lo presencial con lo virtual mediante el uso de nuevas y mejores metodologías que reúnan lo principal de cada una para ofrecer una educación de calidad (Cano, 2015). Esta modalidad educativa contribuye a una participación más colaborativa entre estudiantes. Éstos se inspiran y despiertan habilidades tales como la creatividad y el deseo de innovar, habilidades que, aunque inherentes a ellos, no les permitían emerger por falta de impulso y motivación.

El cambio postmoderno, aunado al b-learning, desempeña un papel muy importante en la práctica docente, al mostrar la conjugación de las bondades de la formación presencial con las funcionalidades del e-learning, lo que lleva a fortalecer el proceso de enseñanza-aprendizaje. Este cambio se convierte en un desafío para el docente, porque éste facilita e impulsa la participación no sólo individual sino también colaborativa, transformando su papel de docente tradicional en un proceso co-constructivista, aunado a diversas experiencias en el aula, que promueve la autonomía del estudiante y le cede a éste el protagonismo en su proceso de enseñanza-aprendizaje.

Las estrategias de enseñanza-aprendizaje han generado un cambio radical en los últimos años, haciendo frente a muchos desafíos de dinámicas en las que el aprendizaje se envuelve de reflexión, autonomía y co-construcción de conocimiento, donde los estudiantes que problematizan situaciones las resuelven al crear espacios de co-colaboración e intercambio 
de saberes. El pensamiento crítico-reflexivo-creativo puede dirigir su camino profesional de manera más asertiva posteriormente.

\section{Revisión bibiliográfica}

Para la presente investigación se hizo una revisión documental en diferentes bases de datos, en el área de educación y bajo la categoría de b-learning. La revisión de estas investigaciones evidencia la importancia de integrar en la educación superior esta modalidad educativa: "La transformación universitaria es una necesidad urgente, que debe asumir varios cambios y retos dentro de un mundo a menudo más cambiante y dinámico" (González, 2015).

El análisis sobre el proceso de adopción de las innovaciones educativas ha sido realizado desde diferentes perspectivas, pero se asume que, en general, se produce mediante una secuencia de aceptación de ellas, que se da de manera gradual y por etapas. Según esto, los profesores pasan de un estado de mínimo contacto con la innovación a uno final en el que el propósito es su integración normalizada en la práctica cotidiana docente (Martín García et al., 2019). Se han realizado diversos estudios relacionados con el proceso de enseñanzaaprendizaje respecto al b-learning, como es el caso de Bartolomé (2004), Gebera y Washington (2010) y Muñoz-Repiso y Tejedor (2017), los que confirman que el alumno valora con claridad la incidencia de las TIC en el desarrollo de estrategias eficaces en el proceso de enseñanza-aprendizaje. Además, "el aprendizaje combinado ofrece una solución a las limitaciones de espacio y tiempo de lecciones presenciales" (Graham, 2006).

Los metanálisis también demuestran claramente la efectividad del aprendizaje combinado en el rendimiento de los estudiantes, en comparación con el aprendizaje en línea y el aprendizaje presencial (Batdı, 2014; Çırak Kurt et al., 2018). Por su parte, Graham et al. (2014) y Staker y Horn (2012) aportan tipologías o modelos de b-learning que responden a criterios de distribución de las actividades entre el entorno presencial y a distancia. Como resultado de su investigación, Çırak (2016) identificó nueve componentes efectivos para el aprendizaje combinado, como roles de docentes, actividades en el diseño, sistema de gestión de aprendizaje [learning management system, LMS], lecciones presenciales, materiales del curso en línea, interacción entre estudiantes, valoración y evaluaciones, roles de los alumnos, y uso compartido en línea.

Respecto a investigaciones realizadas sobre la percepción de los docentes al hacer uso de b-learning en el proceso de enseñanza-aprendizaje, el trabajo de Martín García et al., (2019) que hace referencia a un estudio donde se muestra la percepción del b-learning en profesores universitarios de distintos ámbitos disciplinares, indica una elevada aceptación entre los docentes de las ventajas de utilización de entornos basados en esta modalidad. Sin embargo, Martín García y Sánchez Gómez (2014), en su investigación sobre el modelo predictivo de la intención de adopción del b-learning entre profesores universitarios, mencionan que muchos docentes conocen esta modalidad formativa pero, o bien no son plenos usuarios de ella, 
o bien trasladan parte de su rutina docente, basada en la clase presencial, al formato e-learning, sin la necesaria integración equilibrada de ambas modalidades.

Por otra parte, González Fernández (2018), en su estudio respecto a la percepción del desempeño docente-estudiante en la modalidad mixta desde una mirada ecosistémica, demuestra que los docentes reconocen que, a partir de sus competencias, logran que el estudiante mejore en los siguientes aspectos: planeación, gestión del ambiente de aprendizaje y evaluación promotora de la autogestión.

La revolución de las TIC implica nuevas concepciones del proceso de enseñanza-aprendizaje; a partir de ellas, la universidad apuesta al cambio para cumplir su misión y visión, sistematizando e identificando los mecanismos que intervienen en el proceso de enseñanzaaprendizaje.

Este entorno de globalización en el que se inserta la universidad ha ido configurando nuevas oportunidades para la educación superior, que está experimentando continuamente cambios que representan oportunidades y desafíos, ante la demandante sociedad que ahora exige un nuevo perfil del estudiante, que responda a los nuevos requerimientos del proceso productivo y a las formas de organización del trabajo resultantes de la revolución tecnológica, donde se requieren modelos educativos más flexibles para que la instrucción sea más efectiva y facilite el acceso al camino del aprendizaje sin importar el espacio y tiempo.

\section{Metodología}

A partir del planteamiento general expresado anteriormente se propone desarrollar un proceso de análisis en torno a la siguiente pregunta de investigación: ¿existen diferencias entre los docentes y estudiantes universitarios en la percepción de uso en el aula de la metodología b-learning? Para dar respuesta a esta cuestión y conseguir el objetivo propuesto, este trabajo se ha sustentado en una metodología cualitativa y de carácter interpretativo, que recupera las experiencias del docente respecto a la modalidad educativa $b$-learning y su inclusión en su quehacer educativo en el aula, así como la percepción del alumnado respecto a las potencialidades de esta modalidad y a su capacidad para generar un cambio e innovación en el proceso de enseñanza-aprendizaje.

\section{Participantes}

En el estudio participó un total de 54 docentes y 120 estudiantes, todos activos. En cuanto al género, 24 (44.4\%) eran mujeres y 30 (55.6\%) hombres. La distribucuión por edad osciló entre 32 y 46 años, los cuales suman más del 7\% de la muestra. Los profesores se encuentran adscritos a los departamentos de Ciencias Básicas y Ciencias Tecnológicas, e imparten clases en las áreas de Ingeniería en Computación e Ingeniería en Informática, del Centro Universitario de La Ciénaga (CUCI) de la Universidad de Guadalajara (UDG). 
Por su parte, el alumnado se compone de más hombres que mujeres (58.3\% y $41.7 \%$ respectivamente), la mayoría menores de 22 años. El 62\% señaló haber utilizado las plataformas virtuales en el proceso de enseñanza-aprendizaje. Los participantes fueron informados sobre los propósitos de la investigación, y aceptaron libre y voluntariamente formar parte del estudio.

\section{Diseño, procedimiento, instrumento y análisis de datos}

Se seleccionó como instrumento de investigación para la recolección de datos dos cuestionarios paralelos, con un diseño transversal y dimensiones distintas, de acuerdo con los participantes en la investigación, que fueron organizados en tres secciones. La primera se enfocó en los datos generales; la segunda hace referencia a la percepción de los docentes-alumnos con respecto al proceso propio de enseñanza-aprendizaje, mediante la utilización de las herramientas del b-learning, y la tercera se refiere a la aplicabilidad de los recursos de las diferentes plataformas virtuales en el quehacer docente, con un total de veinte preguntas.

\section{Experiencias del docente}

El factor principal es que los docentes se resisten a cambiar su práctica académica, porque es un giro difícil para aquellos cuya construcción del conocimiento ha sido tradicionalista. En esta línea de investigación se realizó un estudio cuyo objetivo fue conocer la experiencia del docente utilizando las herramientas pedagógicas que proporciona el b-learning como apoyo en el aula. Se ha focalizado la formación del docente en estas herramientas digitales. Ha predominado la forma como éste fue formado profesionalmente, no obstante se han diseñado iniciativas para promover el cambio a través de cursos curriculares orientados a esta misma temática. De acuerdo con el resultado de las encuestas, la percepción de los profesores es esencial para que el uso y apropiación de modalidades educativas y la cohesión de éstas con la práctica docente sea más efectiva, debido a que la formación del futuro, según el nivel educativo al que se hace referencia en este trabajo, se caracteriza por una serie de hechos. Se plantea el $b$-learning como una alternativa que pretende salvar las limitaciones de las soluciones informatizadas para el autodesarrollo. Como es sabido, este paradigma se fundamenta en el empleo de diferentes estrategias de aprendizaje, mediante el aprovechamiento de las virtudes de cada una de ellas, lo cual posibilita el desarrollo integral del alumno (Arranz y Aguado, 2005).

\section{Resultados}

Profesor

En esta sección se describe la experiencia de los docentes de los programas educativos de Ingeniería en Informática e Ingeniería en Computación, con respecto al uso de los procesos de $b$-learning en la enseñanza-aprendizaje en el aula. Los ambientes virtuales son herramientas

ISSN: 2007-1760 (impreso) 2448-9026 (digital) | DOI: 10.30973/inventio/2021.17.41/5 
dinámicas que nos ofrecen comunicación sincrónica y asincrónica. La incorporación de estas tecnologías en la docencia conlleva una nueva modalidad del proceso de enseñanza-aprendizaje.

En la tabla 1 se observa que el docente considera que emigrar a un modelo educativo que implica el uso del $b$-learning supone más trabajo y esfuerzo para el profesor (75.4\%) y el $73.6 \%$, que se requiere formación en nuevas estrategias de aprendizaje para orientar a los estudiantes sobre el uso adecuado de los recursos tecnológicos. Sin embargo, un menor número $(56.1 \%)$ piensa que es necesario que tanto el profesor como el estudiante estén convencidos de su nuevo papel en el proceso de enseñanza-aprendizaje, y que cambien su rol como docentes y estudiantes, respectivamente.

Tabla 1

Experiencias del profesorado relacionadas

con el paradigma presencial optimizado

¿Qué piensa respecto a las nuevas estrategias de aprendizaje (paradigma presencial optimizado)?

La orientación a los estudiantes sobre el uso correcto de los recursos tecnológicos dentro $\quad 73.6 \%$ del aula y fuera de ella.

La adaptación del nuevo rol del docente y del estudiante. $\quad 56.1 \%$

Generará más trabajo y esfuerzo para el profesor.

$75.4 \%$

Respecto a la repercusión de una modalidad híbrida en los procesos de enseñanza-aprendizaje, el $82.6 \%$ de los profesores consideran que surgen nuevas formas de co-construir el conocimiento con un enfoque colaborativo y el 91.4\% afirma que la inserción de la tecnología en el aula contribuye a la autonomía de los estudiantes en su recorrido educativo-formativo sin tiempo ni espacio determinado, lo que implica el fortalecimiento en el desarrollo de otras potencialidades (tabla 2).

Tabla 2

Influencia de una modalidad híbrida en los procesos de enseñanza-aprendizaje ¿De qué forma influye la inserción de una modalidad híbrida en los procesos de enseñanza-aprendizaje? Nuevas formas de aprender. $\quad 82.6 \%$

Trabajo autónomo de los estudiantes en una educación sin tiempo ni espacio determinado. $\quad 91.4 \%$

Estos hallazgos coinciden con el estudio de Carranza Alcántar (2017), quien afirma que los docentes reconocen la complementariedad que esta modalidad implica, en la cual se puede hacer uso de la tecnología y reforzar conocimientos en las clases presenciales. En opinión de $77.6 \%$ de los profesores, la modalidad del $b$-learning incide en la diversidad en el proceso de enseñanza-aprendizaje y supone facilidad de acceso cada vez más generalizada. El 81.1\%

Año 17, núm. 41, marzo 2021

ISSN: 2007-1760 (impreso) 2448-9026 (digital) | DOI: 10.30973/inventio/2021.17.41/5 
de los profesores considera que la flexibilidad que hoy en día ofrecen las tecnologías permite la combinación de metodologías, toda vez que el proceso tecnológico y social de cambio actual, así como los procesos de cambio e innovación que se están dando en la educación, inducen al desarrollo de un cambio trascendental en los estudiantes. El 85.4\% afirma que el uso del $b$-learning influye en el desarrollo de habilidades de pensar y actuar (tabla 3).

\section{Tabla 3}

\begin{tabular}{lc}
\multicolumn{2}{c}{ Uso del b-learning } \\
\hline ¿Qué ventajas encuentra en vivir el proceso de alineación utilizando b-learning? \\
\hline Diversidad del proceso de enseñanza aprendizaje. & $77.6 \%$ \\
La flexibilidad de las tecnologías permite combinar las metodologías, al desarrollar una & $81.1 \%$ \\
transformación trascendental en los estudiantes. & \\
Habilidades de pensar y actuar. & $85.4 \%$ \\
\hline
\end{tabular}

Los docentes han utilizado diferentes estrategias para que el estudiante desarrolle el pensamiento crítico mediante un aprendizaje significativo-colaborativo. El $85.4 \%$ ha utilizado proyectos basados en problemas; el 81.1\% afirma que le ha funcionado la estrategia del aprendizaje cooperativo, y al 57.2\% le ha gustado trabajar con lecturas y videos (tabla 4).

\section{Tabla 4}

Estrategias de aprendizaje $b$-learning

\begin{tabular}{ll}
\hline ¿Cuáles estrategias didácticas de aprendizaje b-learning utiliza con mayor frecuencia? & \\
\hline Proyectos basados en solución de problemas, retos críticos y fomento de la participación. & $85.4 \%$ \\
Aprendizaje cooperativo con plena libertad para los estudiantes. & $81.1 \%$ \\
Lecturas, videos. & $57.2 \%$ \\
\hline
\end{tabular}

El uso de diferentes estrategias en el proceso de enseñanza-aprendizaje en la modalidad mixta se encuentra también en consonancia con el estudio de Carranza Alcántar y Caldera Montes (2018), quienes afirman que la efectividad de las tecnologías depende de la pertinencia de las estrategias utilizadas con respecto al tipo de aprendizaje a desarrollar, es decir, la selección de estrategias mediadas con tecnología debe considerar de qué manera se quiere influir en el aprendizaje y a partir de ellas determinar qué recursos son los más adecuados para alcanzarlos.

\section{La innovación tecnológica en las modalidades educativas}

Los avances sociotecnológicos han hecho posibles las diferentes modalidades educativas que proliferan hoy en día, ante una constante evolución que ha sobrepasado de manera sorpresiva al ámbito educativo en el contexto de la globalización.

Año 17, núm. 41, marzo 2021

ISSN: 2007-1760 (impreso) 2448-9026 (digital) | DOI: 10.30973/inventio/2021.17.41/5 
El enfoque del aprendizaje a lo largo de la vida no plantea que uno tenga que pasarse toda la vida en la universidad, pero sí sugiere que un individuo deberá comprender el aprendizaje como un proceso continuo y abierto, capaz de combinar la adquisición de conocimientos explícitos (codificados o sistematizados) con aquellos conocimientos tácitos y habilidades blandas que demanda la sociedad global en distintos contextos y etapas de la vida (Cobo Romaní y Moravec, 2011).

Las instituciones educativas se apropian de nuevos modelos educativos, más flexibles y eficaces, que marcan un nuevo hito en la educación y modifican la metodología, en los que el aprendizaje es más reflexivo, autocrítico y personal, lo que genera un panorama educativo donde las diferentes formas de aprender se transforman en un pensamiento más crítico y autónomo. No se trata de desechar lo que ya se tiene o se conoce, sino de aprender también de otras maneras. El b-learning se considera un elemento clave en la comunicación bidireccional, que propicia la interacción personal en el aula entre el docente y el estudiante como medio preferente de enseñanza, por la acción sistemática y conjunta de diversos recursos didácticos y el apoyo tutorial, que proporcionan el aprendizaje autónomo de los estudiantes, además de reforzar la habilidad de la comunicación efectiva a través de las plataformas amigables (González, 2015).

\section{Experiencias del estudiante}

En la actualidad, al estudiante se le ofrece la posibilidad de pensar, razonar y resolver problemas, y se abren todas las puertas para que acceda al conocimiento necesario sin limitación de tiempo o espacio. Es momento de aprovechar la oportunidad para analizar cómo se puede mejorar la docencia para potenciar el aprendizaje del estudiante, en un nuevo contexto social y tecnológico.

\section{Resultados}

Alumno

Al analizar las encuestas realizadas a los alumnos, la experiencia de los estudiantes universitarios se torna cada vez más importante respecto al desarrollo trascendental de su formación. Conocer lo que ellos piensan de esta modalidad híbrida en su proceso de enseñanza-aprendizaje es fundamental para dirigir el proceso de educación de la personalidad y el aprendizaje de los estudiantes, abarcando las dimensiones cognitivas, afectivas, sociales y culturales, de donde surge la calidad y el fortalecimiento de los profesionales en su formación.

El $87.6 \%$ de los estudiantes afirman que sí es efectivo el trabajar con $b$-learning, ya que esta modalidad les permite retroalimentarse en el aula, lo que para ellos hace la combinación 
perfecta con las actividades en la plataforma. El 79.3\% opina que la inserción de esta modalidad híbrida en el aula ha sido acertada debido a que tienen acceso a las actividades en cualquier dispositivo, sin importar el tiempo ni el espacio. El 71.4\% se considera satisfecho de trabajar con esta modalidad por los beneficios obtenidos tanto en el aula virtual como en la clase tradicional (tabla 1a).

\section{Tabla $1 a$}

\section{Percepciones del estudiante respecto a trabajar con el b-learning}

\begin{tabular}{|c|c|}
\hline ¿Es efectiva la inserción de una modalidad híbrida, como el b-learning, en el aula? & \\
\hline $\begin{array}{l}\text { Los estudiantes se benefician de las clases en el aula, lo que conforma la combinación } \\
\text { perfecta. }\end{array}$ & $87.6 \%$ \\
\hline $\begin{array}{l}\text { Tienen acceso a las actividades sin importar tiempo o espacio a través de tabletas y } \\
\text { móviles. }\end{array}$ & $79.3 \%$ \\
\hline Ofrecen beneficios tanto de las aulas virtuales como de las clases tradicionales. & $71.4 \%$ \\
\hline
\end{tabular}

Carranza Alcántar (2017) manifiesta en su investigación que "los alumnos aseguran que ser autogestivos les permite desarrollar la capacidad para recordar lo que ya saben y a su vez relacionarlo con lo que están aprendiendo, tratando de entender los conocimientos adquiridos" (p. 15).

Las estrategias didácticas han sido determinantes para co-construir un conocimiento colaborativo en los discentes. Los docentes han formulado estrategias para vencer esa apatía por parte de los estudiantes de trabajar en equipo, para motivarlos y despertar su interés por el conocimiento y así desarrollar su pensamiento crítico-reflexivo-colaborativo. Al preguntarles a los estudiantes sobre las estrategias que ha utilizado su asesor para desarrollar esta habilidad, el 73.6\% afirma que éstas han contribuido a que se sientan más motivados y tengan una participación más activa en el aula, el 69.3\% dicen ser más autónomos en su aprendizaje y ya no depender de lo que les comparta el asesor, sino que ellos son los protagonistas de su aprendizaje, y el $65.4 \%$ afirma que han adquirido la habilidad de trabajar en equipo y ser más colaborativos (tabla 2a).

Tabla 2a

Estrategias didácticas

¿Te ha parecido útil la estrategia didáctica que ha utilizado tu asesor para desarrollar tu pensamiento crítico reflexivo? ¿Por qué?

\begin{tabular}{ll}
\hline Sí, porque me siento más motivado y participo de una forma más activa. & $73.6 \%$ \\
Sí, porque he desarrollado un aprendizaje más autónomo. & $69.3 \%$ \\
Sí, porque he aprendido a trabajar en equipo. & $65.4 \%$
\end{tabular}

La valoración positiva de las TIC en el desarrollo de estrategias de aprendizaje corrobora el estudio de Domínguez (2009), quien expresa que las tecnologías promueven el 
aprendizaje autónomo, el trabajo colaborativo, el pensamiento creativo, el desarrollo de estrategias de resolución de problemas, el trabajo interactivo, la capacidad de argumentación oral y escrita, y la creación de representaciones no lingüísticas de lo que aprenden los alumnos.

Por otro lado, en este escenario, el profesor-asesor sube a la plataforma conferencias grabadas en video por él o por algún otro profesional, que los estudiantes pueden ver fuera del aula y luego realizar la actividad correspondiente. Este enfoque facilita el aprendizaje al permitir que los estudiantes hagan preguntas a su maestro o colaboren con sus compañeros mientras realizan su trabajo, y evita dificultades en casa o, en su defecto, tener que pedir ayuda al asesor hasta el siguiente día, cuando lo vea en el aula (Arfstrom, 2014).

El video didáctico es muy útil en la clase y tiene una intención motivadora, ya que, más que transmitir información exhaustiva y sistematizada sobre el tema, pretende abrir interrogantes y generar una dinámica participativa, entre otras acciones, como complemento de los aprendizajes realizados, tanto individuales como en las sesiones de clase.

Al preguntarle a los estudiantes al respecto, el $77.3 \%$ considera que los videos propuestos en el aula han sido útiles porque el asesor los complementa, también en el aula, con autoevaluaciones donde ellos pueden recuperar lo aprendido, mientras que el 52.3\% menciona que durante la transmisión del video el asesor interviene para explicarles detalles que pueden no haberles quedado claro y el $37.2 \%$ sólo comenta que los videos educativos son útiles en el proceso de aprendizaje porque son un medio de apoyo (tabla 3a).

Tabla $3 a$

\section{El video educativo como apoyo en el proceso de enseñanza-aprendizaje}

\begin{tabular}{l}
\hline ¿Consideras que los videos propuestos por tu asesor han sido útiles en tu proceso de enseñanza- \\
aprendizaje? ¿Por qué? \\
\hline $\begin{array}{l}\text { Sí, porque siempre están complementados con autoevaluaciones para recuperar lo } \\
\text { aprendido. }\end{array}$ \\
$\begin{array}{l}\text { Sí, porque el asesor interviene durante la transmisión del video para aclarar detalles. } \\
\text { Sí, porque son un medio de apoyo en el proceso de aprendizaje. }\end{array}$ \\
\hline
\end{tabular}

Los crecientes niveles de alfabetización digital entre estudiantes y profesores son el hito donde el video se convierte en el medio de enseñanza dominante en internet. De acuerdo con las respuestas a la pregunta sobre si el video les ha sido útil como estrategia didáctica, se considera que los videos educativos pueden desempeñar un papel en la documentación del entorno individual y en las contribuciones de los estudiantes. Estos resultados coinciden con la investigación realizada por García Matamoros (2014), quien manifiesta que el video didáctico impacta en el aprendizaje, al permitir al estudiante analizar y valorar la realidad a partir de descripciones e imágenes que puede ser imposible vivir o experimentar, además de estimular la búsqueda de nuevos conocimientos, promover la búsqueda de soluciones a situaciones 
y problemas presentados a través del video, y evaluar su conocimiento, habilidades y destrezas al comparar sus comportamientos con los mostrados en el video.

Según el estudio de Quesada Chaves (2015), se logra obtener un mejor interés por la materia en estudio a través de la utilización de metodologías innovadoras que incorporen la tecnología como parte del proceso de enseñanza-aprendizaje. En consecuencia, según la posición constructivista, el conocimiento no es una copia fiel de la realidad, sino una construcción del ser humano. La persona realiza dicha construcción fundamentalmente con los esquemas que ya posee, es decir, con lo que ya construyó en su relación con el medio que le rodea. Este proceso de construcción depende de dos aspectos fundamentales (Bernheim, 2011):

1. De los conocimientos previos o representación que se tenga de la nueva información, o de la actividad o tarea a resolver.

2. De la actividad externa o interna que el aprendiz realice al respecto.

En las comunidades de aprendizaje virtual, los foros son una participación asíncrona que aporta al aprendizaje, el debate, el consenso de ideas y la construcción compartida del conocimiento. Para ello, un factor fundamental es el asesor, quien crea hilos, modera y responde cuando es necesario. Estos foros de discusión productiva dan lugar al desarrollo de nuevas y dinamizadoras interrogantes que contribuyen a ampliar los horizontes de los nuevos conocimientos y a dar luz al tema de reflexión, incluso temas off-topic, es decir, sin ninguna relación.

En este proceso de comunicación e intercambio, el asesor puede observar cómo el grupo construye los nuevos conocimientos de modo autónomo y colaborativo, además de ser el moderador y quien interviene de forma acertada en las diferentes ideas o participaciones y, a su vez, fortaleciendo y dejando abierta la opción de continuar el debate. A partir de la importancia educativa que tienen los foros de discusión en el aula virtual se hizo la siguiente pregunta a los estudiantes: ¿De qué forma consideras que los foros de debate creados por el asesor apoyan en el aula virtual? (tabla 4a).

El $87.1 \%$ de los estudiantes consideran que los foros potencian el trabajo cooperativo y que éste, a su vez, favorece el aprendizaje colaborativo; el 63.6\% considera que la flexibilidad del tiempo en los foros les hace más fácil reflexionar sobre el tema que se está discutiendo, además de poder aportar en las participaciones de los compañeros, mientras que el 54.2\% dice que los foros les facilita el interactuar con los compañeros.

Martha Arango (2003) menciona que los foros académicos propician mecanismos de participación a través de discusiones que, si bien se valen de los argumentos y reflexiones planteadas por los participantes, deben conducir hacia un diálogo pragmático donde la intención última no es persuadir al interlocutor, sino indagar y utilizar el diálogo para el intercambio de pensamientos, ideas y enfoques variados sobre el tema que se esté discutiendo. 
Tabla 4a

Te han sido útiles los foros de debates

\begin{tabular}{|c|c|}
\hline $\begin{array}{l}\text { ¿De qué forma consideras que los foros de debate creados por el asesor apoyan en } \\
\text { el aula virtual? }\end{array}$ & $\%$ \\
\hline Potencian el trabajo de cooperación con los otros y favorecen el aprendizaje colaborativo. & $87.1 \%$ \\
\hline $\begin{array}{l}\text { Con la flexibilidad en tiempo es más sencillo indagar, reflexionar, organizar y volcar las } \\
\text { ideas sobre las opiniones de los demás participantes. }\end{array}$ & $63.6 \%$ \\
\hline Facilitar el proceso de interacción y de comunicación multidireccional. & $54.2 \%$ \\
\hline
\end{tabular}

La dinámica de trabajo en los foros virtuales invita a los participantes a revisar diariamente las actividades y discusiones, lo que implica una mayor dedicación y tiempo para acostumbrarse al componente virtual (Arango, 2003).

De los resultados descritos anteriormente se pone de manifiesto que, según su propia percepción, el alumnado que ha llevado clases utilizando procesos de b-learning ha cambiado la forma de interactuar con sus compañeros, al transformar su trabajo individual en trabajo colaborativo y haber modificado su forma de aprender, protagonizando así su proceso de enseñanza-aprendizaje y adoptando adecuadamente el uso de las herramientas tecnológicas. Esto coincide con el estudio previo de Fëderov (2006) respecto a la utilización de foros de debate, donde muestra que existe un amplio consenso entre los estudiantes sobre el valor del foro virtual como una herramienta didáctica que incide de manera positiva, significativa e integral en el desarrollo de diferentes aspectos del pensamiento de alta calidad.

\section{Análisis y conclusión}

En el entorno de globalización respecto al ámbito educativo existen dos grandes protagonistas, los cuales son los factores más importantes en estos desafíos que presenta la revolución tecnológica: los docentes, por un lado, quienes para lograr calidad e innovación en sus prácticas pedagógicas requieren de una formación en las TIC, ya que éstas ofrecen grandes oportunidades de mejorar la calidad de la educación, así como actualización en competencias profesionales para mejorar la calidad del proceso de enseñanza-aprendizaje; $y$, por otro lado, los estudiantes, quienes muestran una habilidad inherente con el uso de las TIC.

La flexibilidad en el uso de las TIC y redes para la educación superior atiende a la estructuración de contenidos, en tiempos y espacios de comunicación sincrónica y asincrónica, evaluación de pares y otros, lo que disipa un encuadre jerárquico del poder entre quienes deciden y lideran y quienes son liderados, entre los que enseñan y los que aprenden, entre el diseño, la gestión y la evaluación como momentos disociados, entre el costo y el beneficio, que sólo es un análisis económico y no considera el impacto social (Fainholc, 2016). 
El impacto de una modalidad híbrida en el aula depende de qué tan convencido está el asesor de que las TIC son herramientas de apoyo fundamentales para la actual sociedad de la información y el conocimiento. El grupo de profesores del estudio aquí reportado considera importante el uso del b-learning en el aula, pero una gran mayoría declara que hay más trabajo al utilizarlo que al trabajar con la modalidad tradicional a la que están acostumbrados y como fueron formados.

Los resultados de esta investigación, coincidentes con las opiniones de diversos autores, confirman que el alumno valora con positivismo el desarrollo de estrategias de aprendizaje, pues facilitan las tareas académicas, lo que puede convertirse en una de las claves del éxito académico (Arango, 2003; Chiu y Hsiao, 2010; Fëdorov, 2006; Mortis Lozoya et al., 2015; Muñoz-Repiso y Tejedor, 2017; Vernadakis et al., 2012). Si el profesor observa logros de aprendizaje en sus estudiantes al trabajar con esta modalidad, es probable que siga trabajando así; de lo contrario, es probable que renuncie y vuelva a la modalidad tradicional.

En este contexto, urge que las instituciones tomen medidas respecto a una formación híbrida para los profesores, mediante la selección de un asesor empático, con una variedad de habilidades intrapersonales y de autoliderazgo, como la colaboración, el trabajo en equipo, la administración del tiempo y más. Si bien hoy en día es de vital importancia incluirlas dentro de la educación universitaria para hacer de los jóvenes unos profesionales completos, no estaría de más que el asesor seleccionado para capacitar a los profesores respecto a trabajar con una modalidad híbrida cuente con estas habilidades. La tecnología, la globalización los entornos laborales están cambiando la forma en que las personas trabajarán en el futuro. Muchos de los trabajos del pasado están desapareciendo ante nuestros ojos. Entonces, es necesario formar a los profesores y a los estudiantes incluyendo habilidades blandas. Los docentes que reconocen los beneficios potenciales del aprendizaje mixto para el rendimiento de los estudiantes tienen más probabilidades de adoptar la integración de la tecnología y utilizarla para mejorar la enseñanza y el aprendizaje en el aula.

Esta investigación es una primera aproximación respecto a la convicción que tienen los profesores de trabajar con una modalidad híbrida a partir de sus propias concepciones $y$, al mismo tiempo, un acercamiento para descubrir cómo están viviendo esta experiencia los estudiantes. Este estudio propone la necesidad de seguir analizando las concepciones de los docentes y discentes respecto al uso del b-learning y su relación con lo que demandan la sociedad de la información y del conocimiento para hacer cada vez más coherentes su formación y sus habilidades. 


\section{Referencias}

Arango, M. L. (2003). Foros virtuales como estrategia de aprendizaje. Anexo 1. Universidad de los Andes. http://sgpwe.izt.uam.mx/files/users/virtuami/file/ext/practica comunidades actv forosvirtuales.pdf

Arranz, V. y Aguado, D. (2005). Desarrollo de competencias mediante Blended learning: un análisis descriptivo. Pixel-Bit. Revista de Medios y Educación, 26, 79-88. https://doi. org/10.35362/rie3732710

Arfstrom, K. M. (2014). What's the difference between a Flipped Classroom and Flipped Learning? Ed Tech Focus On K-12. https://www.edtechmagazine.com/k12/article/2014/07/whatsdifference-between-flipped-classroom-and-flipped-learning

Bartolomé, A. (2004). Blended learning. Conceptos básicos. Pixel-bit. Revista de Medios y Educación, 23, 7-20. https://dialnet.unirioja.es/servlet/articulo?codigo=892487

Batdı, V. (2014). The Effect of Blended Learning Environments on Students' Academic Achievement: A Meta-Analysis Study. Journal of Institute of Social Sciences, 5 (1), 287302. https://dergipark.org.tr/en/pub/jiss/issue/25892/272867

Bernheim, C. T. (2011). El constructivismo y el aprendizaje de los estudiantes. Universidades, 48, 21-32. https://www.redalyc.org/pdf/373/37319199005.pdf

Cano, M. G. R. (2015). Impacto del blended learnig en la educación superior. Atenas, 3 (31),5562. https://www.redalyc.org/articulo.oa?id=478047207006

Carranza Alcántar, M. del R. (2017). Enseñanza y aprendizaje significativo en una modalidad mixta: percepciones de docentes y estudiantes. RIDE. Revista Iberoamericana para la Investigación y el Desarrollo Educativo, 8 (15), 898-922. https://doi.org/10.23913/ride. v8i15.326

Carranza Alcántar, M. del R. y Caldera Montes, J. F. (2018). Percepción de los Estudiantes sobre el Aprendizaje Significativo y Estrategias de Enseñanza en el Blended Learning. REICE. Revista Iberoamericana Sobre Calidad, Eficacia y Cambio en Educación, 16 (1). https://doi. org/10.15366/reice2018.16.1.005

Chiu, C. -H. y Hsiao, H. -F. (2010). Group differences in computer supported collaborative learning: Evidence from patterns of Taiwanese students' online communication. Computers and Education, 54 (2), 427-431. https://doi.org/10.1016/j.compedu.2009.08.026

Çırak, S. (2016). An investigation on effectiveness of blended learning supported by Quantum Learning Design Framework [Tesis de Doctorado, Universidad de Gaziantep]. https:// tez.yok.gov.tr/UlusalTezMerkezi/tezDetay.jsp?id=WIWfHwCOhma NYygtgZkDg\&no= X43mrPGHo3GO1ybmFOXpmw

Çırak Kurt, S., Yıldırım, İ. y Cücük, E. (2018). The effects of blended learning on student achievement: A meta-analysis study. Hacettepe University Journal of Education, 33 (3), 776-802. http://doi.org/10.16986/HUJE.2017034685 
Cobo Romaní, C. y Moravec, J. W. (2011). Aprendizaje Invisible. Hacia una nueva ecología de la educación. Laboratori de Mitjans Interactius/Publicacions i Edicions de la Universitat de Barcelona.https://www2.educationfutures.com/books/aprendizajeinvisible/download/ Aprendizajelnvisible.pdf

Domínguez, E. (2009). Las TIC como apoyo al desarrollo de los procesos de pensamiento y la construcción activa de conocimientos. Revista del Instituto de Estudios de Educación, 10, 146-155. http://hdl.handle.net/10584/4615

Fainholc, B. (2016). Presente y futuro latinoamericano de la enseñanza y el aprendizaje en entornos virtuales referidos a educación universitaria. Revista de Educación a Distancia (RED), 48. https://revistas.um.es/red/article/view/253431

Fëdorov, A. N. (2006). Foro virtual como una estrategia metodológica para el desarrollo del pensamiento crítico en la universidad. Innovación Educativa, 6 (30), 62-72. https://www. redalyc.org/articulo.oa?id=179420843006

García Matamoros, M. A. (2014). Uso Instruccional del video didáctico. Revista de Investigación, 38 (81), 43-67. https://www.redalyc.org/articulo.oa?id=376140396002

Gebera, T. y Washington, O. (2010). Contexto y desarrollo de la modalidad educativa blended learning en el sistema universitario iberoamericano. Revista Mexicana de Investigación Educativa, 15 (45), 345-370. https://www.redalyc.org/articulo.oa?id=14012507002

Graham, C. R. (2006). Blended Learning Systems. Definition, current trends and future directions. En J. Curtis, Ch. Bonk y R. Graham (Ed.), The Handbook of Blended Learning: Global Perspectives, Local Designs. John Wiley \& Sons.

Graham, C., Henrie, C. y Gibbons, A. (2014). Developing models and Theory for Blended Learning Research. En A. Picciano, C. Dziuban y C. Graham (Eds.), Research Perspectives in Blended Learning: Research Perspectives, 2 (13-33). Routledge.

González Fernández, M. O. (2018). Percepción del desempeño docente-estudiante en la modalidad mixta desde una mirada ecosistémica. RIDE. Revista Iberoamericana para la Investigación y el Desarrollo Educativo, 8 (16), 370-393. https://doi.org/10.23913/ride. v8i16.346

González, M. E. (2015). El b-learning como modalidad educativa para construir conocimiento. Opción, 31 (2), 501-531. https://www.redalyc.org/articulo.oa?id=31045568029

Martín García, A. V. y Sánchez Gómez, M. C. (2014). Modelo predictivo de la intención de adopción de Blended learning en profesores univeristarios. Universitas Psychologica, 13 (2). http://doi.org/10.11144/Javeriana.UPSY13-2.mpia

Martín García, A.V., Sánchez Gómez, M. C.y Costa, A.P. (2019). Percepción de Blended Learning en profesores universitarios de distintos ámbitos disciplinares. Revista Lusófona de Educação, 44, 117-133. https://www.redalyc.org/articulo.oa?id=34962082009 
Muñoz-Repiso, A. G. V. y Tejedor, F. J. T. (2017). Percepción de los estudiantes sobre el valor de las TIC en sus estrategias de aprendizaje y su relación con el rendimiento. Educación XXI, 20 (2), 137-159. https://www.redalyc.org/articulo.oa?id=70651145006

Mortis Lozoya, S. V., Parra, E. del H., García López, R. I. y Valenzuela, A. M. (2015). La modalidad mixta: un estudio sobre los significados de los estudiantes universitarios. Innovación Educativa, 15 (68), 73-97. http://www.scielo.org.mx/scielo.php?script=sci arttext\&pid=S1665-26732015000200006\&lng=es\&tlng=es

Quesada Chaves, M. J. (2015). Creación de videos educativos como estrategia didáctica para la formación de futuros docentes de inglés. Revista Electrónica Actualidades Investigativas en Educación, 15 (1), 1-19. https://www.redalyc.org/articulo.oa?id=44733027006

Staker, H.y Horn, M. B. (2012). Classifying K-12 Blended Learning. San Mateo: Innosight Institute, Inc. Vernadakis, N., Giannousi, M., Derri, V., Michalopoulos, M. y Kioumourtzoglou, E. (2012). The impact of blended and traditional instruction in students' performance. Procedia Technology, 1, 439-443. https://doi.org/10.1016/j.protcy.2012.02.098 